\title{
MEDICAMENTOS DE VENTA LIBRE: Entre el acceso y el uso inseguro e irracional.
}

Por: Maureen Solano O. Egresada de Farmacia, UCIMED

Marisol Flores C. Coordinadora CEDIFAR y docente, UCIMED Randall Madrigal M. docente, UCIMED

Gustavo Sáenz G. Decano de Farmacia y docente, UCIMED

\section{Se suma esta reflexión a la discusión sobre la idoneidad de ofrecer medicamentos bajo la categoría de venta libre en establecimientos comerciales no especializados.}

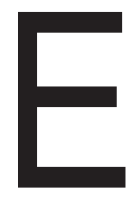

I deseo de abordar los procesos de enfermedad y de prevención de las condiciones infecciosas que pueden llevar a las personas de un país a este fatal desenlace, han sido prioridad desde el inicio del establecimiento de las políticas de Salud de Costa Rica.

Algunos ejemplos de principios del siglo XX, lo podemos identificar con el establecimiento de una campaña contra la anquilostomiasis ${ }^{1}$, lo que llevó quince años después al establecimiento del Departamento de Anquilostomiasis en la Secretaría de Policía ${ }^{2}$. Con el paso del tiempo, el aumento de la preparación y conciencia de la atención de la salud, así como, de los recursos destinados para estos efectos, se crea en 1922 y por Decreto Ejecutivo, la Secretaría de Higiene y de Salud Pública, la cual centraliza algunas iniciativas creadas en años anteriores como el Servicio Antimalárico, de Profilaxis Venérea, las Clínicas de Atención Infantil y Clínicas Prenatales. Y un año más tarde en la Ley No. 72 "Sobre la Salud Pública" (marzo de 1923) se creó la Dirección de los Servicios de Salud Nacionales como dependencia del estado. Consecuentemente, como resultado de una reestructuración

En 1949 con la promulgación por Decreto No. 809 un nuevo Código Sanitario que transforma en la Secretaría en Ministerio. A partir de este momento y en conjunto con la visión de la Organización Mundial de la Salud (OMS) y la acción de algunas otras instituciones especializadas, de Gobierno como, lo que hoy en día es el Instituto de Acueductos y Alcantarillados (AYA), el cual inició como el
Servicio Nacional de Aguas y el establecimiento de la Caja Costarricense de Seguro Social (CCSS), las cuales han generado enormes beneficios a la epidemiología del país, adquiriendo un perfil epidemiológico de países del primer mundo.

Respondiendo a la visión de la OMS, se desarrolla una política de acceso a los medicamentos básicos, la cual queda plasmada en la creación de la CCSS (1973), su cobertura universal, su Lista Oficial de Medicamentos y su enfoque en la atención por niveles, favorece el acceso no solo a la atención de la salud sino también directamente a los medicamentos; lo cual garantiza la coherencia entre lo promulgado por la OMS. Esa misma línea de pensamiento se establece con la creación de una categoría de medicamentos de venta libre, quedando definida de la siguiente manera en la Ley General de Salud (LGS) (art. 120).

Son de venta libre los medicamentos que el Ministerio declare como tales en el correspondiente decreto, oyendo previamente el criterio del Colegio de Farmacéuticos. En el caso de medicamentos para uso veterinario será también

consultado el Colegio de Médicos Veterinarios.

Para orientar el análisis del tema de medicamentos de venta libre se debe hacer en concurso de algunos otros artículos de la misma u otras normas. Así el artículo 101 de esta misma Ley, establece que "La elaboración, manipulación, venta, expendio, suministro y depósito de los medicamentos sólo podrán hacerse en establecimientos farmacéuticos debidamente autorizados y registrados". Como adendum de análisis se debe reconocer que el establecimiento denominado farmacia en el artículo 95 es "aquel que se dedica a la preparación de recetas y al expendio y suministro de directo al público de medicamentos". Además del establecimiento farmacéutico denominado Botiquín como "aquel pequeño establecimiento destinado de forma restringida, únicamente al suministro de medicamentos que

1- Rica HdC. Reseña Histórica de las Instituciones del Sector Salud - Binasss. [Online].; 1997 [cited 2017 Mayo 02. Availablefrom: http://www. binasss.sa.cr/revistas/hospitales/art84.

2- Iván Molina Jiménez SP. Salud Imperial y Educación Popular. La Fundación Rockefeller en Costa Rica Desde una perspectiva centroamericana (1914 - 1921). In Iván Molina Jiménez SP. Educando a Costa Rica: alfabetización popular, formación docente y género (1880 - 1950). San José: Editorial Universidad Estatal a Distancia; 2003. p. 201 - 205. 
el Ministerio autorice, oyendo previamente el criterio del Colegio de Farmacéuticos. En el caso de medicamentos de uso veterinario, además, oír previamente el criterio del Colegio de Médicos Veterinarios".

Según lo analiza el Lic. Randall Madrigal, "Bien puede señalarse, a nuestro juicio, que no existe otra conclusión más que los establecimientos farmacéuticos, claramente definidos en el numeral 95 de la Ley General de Salud, son los únicos establecimientos en los que se puede elaborar, manipular, vender, expender, suministrar y depositar medicamentos, de tal forma que los medicamentos de venta libre - pues no escapan de la categoría de medicamentos - únicamente pueden ser ofrecidos al consumidor en establecimientos farmacéuticos debidamente autorizados para tal fin. Para el caso que nos ocupa, en la farmacia y en el botiquín, de tal forma que libre venta implica que no requieren de receta médica, no así que puedan ser ofrecidos en cualquier establecimiento comercial"3.

Sin embargo, en 1995, como consecuencia del establecimiento de políticas neoliberales en muchos ámbitos nacionales, el Decreto Ejecutivo No. 35595-S de setiembre del 2009, denominado "Declara Medicamentos de Venta Libre al Consumidor", en el que el Poder Ejecutivo reglamenta lo que dispone el ya citado artículo 120 de la LGS, establece en su artículo 1 lo siguiente: "Declárese de venta libre $y$ en consecuencia pueden ofrecerse al consumidor en cualquier establecimiento comercial, los siguientes medicamentos (...)"4.

Sin embargo, y aun sabiendo que el Poder Ejecutivo tiene potestad para reglamentar las leyes, es claro que este órgano se aparta de lo dispuesto por el legislador, ya que no se refiere a establecimientos farmacéuticos, sino que amplía la oferta de medicamentos de venta libre a cualquier establecimiento comercial. Con todo y el grave riesgo que esto conlleva para la salud pública.

\section{El Poder Ejecutivo incurre en un exceso de potestad reglamentaria.}

\section{Medicamentos de Venta Libre y Seguridad del Paciente}

El tema de medicamentos de venta libre guarda estrecha relación con el abordaje de seguridad del paciente, relacionado este con la protección de la Salud y la protección de la vida, derechos constitucionales de obligada tutela por el Estado.

Si bien la misma Organización Panamericana de la Salud (OPS) establece como Medicamento de Venta sin Receta 5 :

\section{"Producto farmacéutico, medicamento o especialidad medicinal cuya dispensación o administración no requiere autorización médica, utilizados por los consumidores bajo su propia iniciativa y responsabilidad para prevenir, aliviar o tratar sintomas o enfermedades leves y que su uso, en la forma, condiciones y dosis autorizadas sean seguras para el consumidor".}

Es necesario detallar en algunas de las descripciones finales de dicha definición y que se supone deben cumplirse para los productos de esta categoría, para comprender la seriedad de esta categoría.

Condiciones autorizadas: Las condiciones autorizadas son aquellas que, de acuerdo a la norma nacional y que están establecidas en el Reglamento de buenas prácticas de almacenamiento y distribución de medicamentos en droguerías , refieren a los siguientes factores: condiciones de calidad del producto, condiciones de almacenamiento, contaminación, estudios de mapeo de temperatura y humedad, Falsificación de productos, fecha de expira. Estas condiciones se plantean como objetivo de la norma "(...) garantizar que la calidad de los mismos se mantenga y así contribuir a la salud de la población" 6 .

Estas condiciones son tema en gran medida del análisis que se desea proponer puesto que especialmente en estas condiciones es dónde existen algunas inconsistencias de cumplimiento en la cadena logística de distribución de medicamentos de Venta Libre hacia los demás establecimientos comerciales al detalle que no son Farmacias, pues a los distribuidores que venden productos a pulperías, supermercados y bares, no se les exige el cumplimiento de condiciones que garantizan, de acuerdo a lo establecido en el Reglamento de buenas prácticas de almacenamiento y distribución, la seguridad de almacenamiento, ni demás condiciones contra la contaminación, la garantía de la humedad y la temperatura adecuadas.

\footnotetext{
3- Madrigal M R. El Decreto Ejecutivo No. 35595-S y las implicaciones jurídico-sanitarias de los medicamentos de libre venta en Costa Rica. Cad. Ibero-Amer. Dir. Sanit., Brasília, v.3, n.1, jan/jun. 2014

4- COSTA RICA. Decreto Ejecutivo No. 35595-S “Declara Medicamentos de Venta Libre al Consumidor”. 2009.

5- OPS. Grupo de trabajo de clasificación de medicamentos (GT/CM).PDF http://www1.paho.org/spanish/ad/ths/ev/CM-DefinicionycriteriosMLVEs. pdf?ua=1.

6- Costa Rica. Decreto Ejecutivo No. 37700. "Reglamento de buenas prácticas de almacenamiento y distribución de medicamentos en droguerías”. 2013
} 
Seguridad para el consumidor: El mismo documento de la OPS, expresa como criterios para la clasificación de Medicamento de Venta Libre sin Receta:

1.Medicamentos eficaces y seguros para ser utilizados en la prevención, alivio de síntomas o tratamiento de enfermedades leves, de fácil identificación.

2.Medicamentos con amplio rango de seguridad, de tal modo que la administración voluntaria o involuntaria de dosis superiores a las recomendadas o donde no este indicado, no represente un peligro grave para la salud del paciente.

3.Tengan un margen de dosificación amplio, susceptible de ser adaptado a la edad y peso del paciente.

4.Medicamentos cuyo empleo no genere tolerancia o dependencia y que no sean susceptibles de abuso.

5.Cuando se utilice de acuerdo a las instrucciones no enmascaren enfermedades serias, ni retrasen el diagnóstico y tratamiento de una condición que requiere de atención médica.

6.Medicamentos de empleo seguro en todos los grupos de edad de la población.

7.Formas farmacéuticas generalmente de vía oral o tópica, de fácil manejo y almacenamiento y que no sean de administración IV o IM.

8.El principio activo, haya sido comercializado bajo prescripción médica por lo menos 5-10 años, tiempo durante el cual ha demostrado un índice favorable de seguridad y eficacia con datos de farmacovigilancia,

9.Los reportes de reacciones adversas no se hayan incrementado durante el periodo de comercialización.

Si bien existe en el país normativa que establece un Sistema Nacional de Farmacovigilancia ${ }^{7}$, la información sobre seguridad de estos medicamentos, no permite respaldar algunas de las afirmaciones anteriores para establecer los criterios de la OPS, especialmente las dirigidas a garantizar la seguridad en uso de estos medicamentos.
En Costa Rica no existen condiciones para la salvaguarda de las condiciones de almacenamiento y distribución de los medicamentos que se venden en la modalidad de venta libre; ni existe el respaldo de información de seguridad que permita garantizar los criterios establecidos por la OPS.

\section{ACCESO RACIONAL O IRRACIONAL}

La CCSS cubre a más del $94 \%$ de la población con la diversidad de servicios a los que accede la población mediante los Equipos Básicos de Atención Integral de la Salud (EBAIS), el despacho de medicamentos ha alcanzado para el año 2016 poco más de 82 millones de tratamientos despachados a través de las farmacias de la institución, según los informes estadísticos de la CCSS.

Esta cantidad de tratamientos despachados por la Seguridad Social, para una población de 4.5 millones de habitantes aproximadamente (2016), representa poco más de 18 tratamientos en promedio para cada habitante del país.

Si sumamos los más de 20 millones de tratamientos vendidos a través del mercado privado ${ }^{8}$, significa que, en el año 2016 , más de 100 millones de tratamientos medicamentosos fueron entregados a muchas personas en el país.

A pesar de esta realidad y ante consulta del Colegio de Farmacéuticos de Costa Rica que la Procuraduría General de la República emite en 2009, sendos criterios en respuesta a la advertencia de riesgos a la Salud Pública, expresando:

"Frente a la alegación de seguridad de la dispensa de medicamentos se opone la accesibilidad misma del medicamento. En la medida en que determinados medicamentos que no requieren receta médica y no sean susceptibles de provocar riesgos para la salud puedan ser adquiridos en establecimientos comerciales distintos de los farmacéuticos, se garantiza la accesibilidad de la población a los medicamentos. Aspecto que cobra particular importancia en los lugares alejados de los centros de población e incluso en las poblaciones pequeñas en que no está asegurada la permanencia de una farmacia abierta que pueda vender los medicamentos.

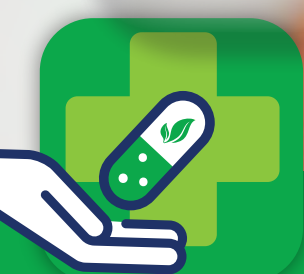


En ese sentido, la imposibilidad jurídica de obtener determinados medicamentos puede implicar que el Estado no brinde la atención y protección adecuada a la salud $y$, antes bien, que llegue a afectar con su prohibición el derecho a la salud. Es decir, en casos como los indicados en los que las personas no tienen un acceso cercano a los establecimientos farmacéuticos, la prohibición absoluta de venta o expendio de medicamentos de bajo riesgo en lugares comerciales bien podría constituirse en un atentado contra la salud de la persona. [... ${ }^{9}$

Se debe garantizar el acceso de la población a los medicamentos y este acceso debe contemplar además las condiciones adecuadas que garanticen la calidad y la seguridad de la población ante el uso de estas herramientas terapéuticas.

\section{PROCESO DE MODIFICACIÓN DE MODALIDAD DE VENTA}

En la actualidad, el Ministerio de Salud requiere del cumplimiento de una serie de pasos para el proceso de modificación de la modalidad de venta de medicamentos de prescripción a la modalidad de venta libre ${ }^{10}$.

Luego de que el interesado entrega el formulario en la Dirección de Atención al Cliente y si esta cumple con lo establecido por una guía de verificación inicial, la información es revisada por un equipo evaluador y es solicitado el criterio de las siguientes entidades:

\section{Colegio de Farmacéuticos de Costa Rica}

Criterios de epidemiología y de seguridad a las asociaciones de médicos especialistas que tengan relación con el medicamento.

\section{Centro Nacional de Farmacovigilancia \\ Centro Nacional de Control de Intoxicaciones}

Algunas consideraciones del proceso identificadas en la investigación referida al pie de la página, revelan varios hechos:
- La solicitud de modificación no detalla en qué indicación aprobada se solicita la modificación, lo cual es de gran importancia para los medicamentos que tienen varias indicaciones aprobadas y a su vez representa un riesgo para la población que no cuenta con el criterio para determinar si su condición puede ser tratada con ese medicamento y en qué dosificación. Ante este hecho, se hace necesario que la información contenida en los medicamentos de venta libre debe ser acorde con el Reglamento Centroamericano de etiquetado para medicamentos de uso en humanos ${ }^{11}$.

- La constitución del grupo evaluador debería contemplar una semejanza a la constitución del Consejo Técnico de Inscripciones, con la participación de los sectores representados de manera que la evaluación sea 10 más integral posible.

- La lista de medicamentos de venta libre no existe de manera consolidada en ningún formato y, por tanto, no está disponible en el sitio web de Ministerio de Salud.

Se demuestra que el proceso de cambio de modalidad de venta es débil técnicamente y no accede a información científica para la adecuada toma de decisiones.

Esta revisión de estado de la cuestión, respecto de los medicamentos de Venta Libre, pretende apoyar a los involucrados a entender el entorno en el que se mueven los medicamentos de venta libre y su proceso de autorización de cambio de modalidad de venta y los antecedentes normativos que han favorecido que en la actualidad una gran cantidad de medicamentos se vendan fuera de los establecimientos farmacéuticos.

El Centro Nacional de Control de Intoxicaciones, ha reiterado año a año en sus informes que el acetaminofén es el medicamento que más intoxicaciones genera en el país, seguido de los medicamentos combinados para síntomas de la gripe, ambos con modalidad de venta libre.

No existen datos duros que nos permitan demostrar la correlación que sugerimos; sin embargo, se hace necesario continuar con la discusión y la optimización de los procesos para que se conviertan en garantes para la población de seguridad y calidad en el acceso a los medicamentos.

9- Costa Rica. Procuraduría General de la República. Dictamen C-131-2011 del 16 de junio de 2011

10- Solano, M. Criterios científicos técnicos utilizados por el Ministerio de Salud de Costa Rica para la aprobación de una Modificación de Modalidad de Venta de un Medicamento. Trabajo Final de Graduación. UCIMED. 2017.

11- COMIECO-LXVII. "Reglamento Técnico Centroamericano RTCA. 11.01.02:04 Productos Farmacéuticos. Etiquetado de Productos Farmacéuticos para uso Humano".https://www.imprentanacional.go.cr/pub/2014/10/17/ALCA56_17_10_2014.pdfpara uso Humano".https://www.imprentanacional.go.cr/pub/2014/10/17/ALCA56_17_10_2014.pdf 